\title{
Transatlantica
}

Revue d'études américaines. American Studies Journal

\section{Une exposition en blanc}

La modernité comme absence dans l'art américain, de Winslow Homer à Robert Ryman

\section{Sophie Lévy}

\section{(2) OpenEdition}

1 Journals

Édition électronique

URL : http://journals.openedition.org/transatlantica/313

DOI : $10.4000 /$ transatlantica.313

ISSN : $1765-2766$

Éditeur

AFEA

\section{Référence électronique}

Sophie Lévy, "Une exposition en blanc », Transatlantica [En ligne], 1 | 2005, mis en ligne le 26 mars 2006, consulté le 29 avril 2021. URL : http://journals.openedition.org/transatlantica/313 ; DOI : https:// doi.org/10.4000/transatlantica.313

Ce document a été généré automatiquement le 29 avril 2021.

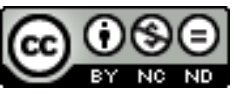

Transatlantica - Revue d'études américaines est mis à disposition selon les termes de la licence Creative Commons Attribution - Pas d'Utilisation Commerciale - Pas de Modification 4.0 International. 


\title{
Une exposition en blanc
}

\author{
La modernité comme absence dans l'art américain, de Winslow Homer à \\ Robert Ryman
}

\section{Sophie Lévy}

1 Cette présentation est celle d'une exposition qui n'existe pas sur l'importance du blanc dans la peinture moderne américaine, et des enjeux historiques et théoriques qui pourraient la soutenir. Il est toujours difficile de parler d'une exposition qui n'existe pas, c'est-à-dire essayer de donner une structure verbale à un exercice visuel, et phénoménologique sans corps. L'expérience, déjà tentée, permet néanmoins de clarifier pour soi-même le sens du projet et de mesurer une réception possible.

2 L'exercice est d'autant plus difficile pour ce projet que les expositions thématiques font entrer en tension le discours et les œuvres, l'un contraignant les autres, les appauvrissant. D'autre part, les thèmes qui s'appuient sur des rapprochement esthétiques peuvent être, presque systématiquement, accusés de superficialité ou d'anachronisme, puisqu'elles affirment la domination de la mise en rapport sur les œuvres individuelles, du maintenant sur l'hier. Enfin les œuvres blanches sont impossibles à reproduire, et l'on ne peut les expérimenter que dans leur réalité de surface, de limites, de couleur, d'agencement.

3 Deux présentations de ce projet virtuel sont d'ailleurs possibles, l'une chronologique, fait apparaître les œuvres au moment de leur création, en se contentant de réfléchir aux charnières temporelles, qui servent de structuration du projet. C'est l'organisation que l'on pourrait préférer lors d'une exposition, parce qu'elle donne une plus grande liberté aux œuvres, sans faire uniquement dépendre leur apparition soit de l'art de l'accrochage, soit du panneau pédagogique.

I.

4 Voici donc ce qu'un tel agencement montrerait :

James McNeill Whistler

5 1. Symphony in White, No. 1: The White Girl, 1862, oil on canvas, Washington, D.C., National Gallery of Art, http://artchive.com/artchive/w/whistler/whistler_white_girl.jpg.html 
6 2. Symphony in White, No.2: The Little White Girl, 1864, oil on canvas, 76.5 x $51.1 \mathrm{~cm}$ London,TateGallery, http://www.artchive.com/artchive/w/whistler/whistler_symphony_white2.jpg.html

7 3. Symphony in White, No.3, 1865-1867, oil on canvas, $52 \times 76.5 \mathrm{~cm}$, The University of Birmingham, The Barber University of Fine Arts, http://www.abcgallery.com/W/whistler/whistler30.html John Singer Sargent

8 4. Staircase in Capri, 1878, oil on canvas, $81.9 \times 46.3 \mathrm{~cm}$, London, private collection http://www.jssgallery.org/Paintings/Staircase_in_Capri.htm

9 5. A Parisian Beggar Girl, c. 1880, oil on canvas, 64.5 x $43.7 \mathrm{~cm}$, Chicago, Terra Foundation for American Art http://www.terraamericanart.org/collections/code/emuseum.asp?

style=Browse\&currentrecord=1\&page=search\&profile=objects\&searchdesc=sargent\&newvalues=1\&newstyle=single\&newc 6. Fumée d'ambre gris, 1880, oil on canvas, 139.1 x $90.8 \mathrm{~cm}$, Williamstown (MA), Clarke Art Institute http://www.jssgallery.org/Paintings/Smoke.htm

11 7. Garden Study of the Vickers Children, 1884, oil on canvas, 137.6 x $91.1 \mathrm{~cm}$, Flint (MI) Institute of Arts http://www.jssgallery.org/Paintings/Garden_Study_of_the_Vickers_Children.htm 8. Lord Dalhousie, 1900, oil on canvas, $152.4 \times 101.6 \mathrm{~cm}$, private collection http://www.artrenewal.org/asp/database/art.asp?aid=187 9. Lights and Shadows, Corfu, 1909, watercolor, $61 \times 76 \mathrm{~cm}$, Boston, Museum of Fine Arts http://www.jssgallery.org/Paintings/Lights_and_Shadows_Corfu.htm

14 10. Two Girls in White Dresses, 1909-1911, oil on canvas, $271 / 2$ x 21 1/2 in., private collection http://www.jssgallery.org/Paintings/Two_Girls_in_White_Dresses.htm Gallery of Art, Smithsonian Institution http://www.jssgallery.org/Paintings/Breakfast_In_The_Loggia.htm John Henry Twatchman 12. Winter Landscape, 1890-1900, oil on canvas, 76.5 x $76.5 \mathrm{~cm}$, Chicago, Terra Foundation for American Art http://www.terraamericanart.org/collections/code/emuseum.asp? style=Browse\&currentrecord=1\&page=search\&profile=objects\&searchdesc=twachtman\&newvalues=1\&newstyle=single\&n Winslow Homer

17 13. Fox Hunt, 1893, oil on canvas, 96.6 x $174.2 \mathrm{~cm}$, Philadelphia, Pennsylvania Academy of Fine Arts http://www.artchive.com/artchive/H/homer/fox_hunt.jpg.html Mary Cassatt

18 14.Mother and Child Against a Green Background (Maternity), 1897, pastel sur papier monté sur toile, Paris, Musée d'Orsay http://www.ibiblio.org/wm/paint/auth/cassatt/maternity.jpg Georgia O'Keeffe

19 15. Morning Glory with Black, No.3, 1926, oil on canvas, $91 . \mathrm{cm} \times 75.5 \mathrm{~cm}$, Cleveland (OH) Museum of Art 
http://www.clevelandart.org/Explore/artistwork.asp?

artistLetter $=0 \&$ recNo $=0 \&$ woRecNo $=0 \& v i e w=$ more

16. Closed Clam Shell, 1926, oil on canvas, $50.8 \times 22.9 \mathrm{~cm}$, private collection

http://www.arts-studio.com/cgi-bin/shop/okeeffe.cgi?page=9

Cow's Skull with Calico Roses, 1932, oil on canvas, 91 x $61 \mathrm{~cm}$, Chicago, Art Institute

http://pavlov.psyc.queensu.ca/ psyc382/okefskul.html

Charles Sheeler

17. Upper Deck, 1929, oil on canvas, $73 \mathrm{~cm} \times 55.3 \mathrm{~cm}$, Cambridge (MA), Fogg Art Museum

http://www.artmuseums.harvard.edu/collections/servlet/

webpublisher.WebCommunication?

ia =codetail\&ic=basic\&oid=861\&sq=1\&tr=1\&tech=\&name=sheeler\&title=upper\%20deck\&objtype=\&medspt=\&dtfrom=\&dtto-

Sam Francis

18. Other White, 1952, oil on canvas, 205,5 x $190,5 \mathrm{~cm}$, Paris, Musée national d'Art moderne

http://tinguely.cnac-gp.fr/image_single.php?

maxh=738\&is_sel=0\&aid=677\&ord=title\&sl=FRANCIS\&iid=14448\&it=2\&expand=0\&cc=-1

Robert Rauschenberg

19. Erased De Kooning Drawing, 1953, work on paper, 64 x 55 cm, San Francisco MoMA

http://www.sfmoma.org/msoma/artworks/93.html

20. White Paintings, 1952

http://www.guggenheim.org/exhibitions/singular_forms/highlights_1a.html

Barnett Newman

21. The Name II, 1950, magna and oil on canvas, $104 \times 94.5 \mathrm{~cm}$, Washington, National Gallery of Art

http://www.nga.gov/cgi-bin/pimage?69323+0+0

Agnes Martin

22. Untitled de 1973 à 2003

http://www.diacenter.org/exhibs_b/martin/

http://members.aol.com/mindwebart4/agnes2.htm

Robert Ryman

http://www.artnet.com/ag/fineartthumbnails.asp?aid=14749

http://www.pacewildenstein.com/jsp/PressReleaseDetail.jsp?

pageType $=$ exhibit\&pressID $=1059 \&$ selectedWorkID $=393 \&$ startRow $=0$

II.

La deuxième démarche vise à dégager des problématiques qui sous-tendent le rapprochement des concepts de blancheur, de modernité et d'art américain. C'est cette approche que nous avons choisie ici, en une importante concession au format de la conférence.

Trois questions nous ont semblé découler du projet :

- le blanc comme absence moderne, ou stade ultime de la logique moderniste théorisée par Clement Greenberg

- le blanc comme déracinement, absence de lieu, cosmopolitisme, nation flottante et de ce fait, infini lieu de projection

- le blanc comme pureté, puritanisme, racisme. 
« Less is more »

31 La géographie de l'art blanc suit celle de la modernité et de l'abstraction. Au XIXe siècle, elle est à Paris. C'est ainsi que James Whistler utilise le blanc, dans cette œuvre (fig.3), aussi comme moyen de composition, qui accentue la frontalité décorative de la toile. Il faut noter que le titre de Symphonie, qui fait penser à la musique, correspond plus à une justification du caractère abstrait de l'œuvre.

Pendant la première moitié du XXe siècle, elle est en Russie, aux Pays-Bas, en Allemagne. On peut par exemple citer ce que dit Wassily Kandinsky, dans Du Spirituel dans l'Art, du blanc:

Le Blanc apparaît comme le symbole d'un monde d'où toutes les couleurs, en tant que propriétés matérielles et substances, auraient disparu. Ce monde est tellement au-dessus de nous qu'aucun son ne nous en parvient. [...] Le blanc agit sur notre âme (psyché) comme un grand silence, absolu pour nous. Pourtant, c'est un silence qui n'est pas mort, mais plein de possibilités. [...] C'est un néant qui est jeune, ou encore plus exactement, un néant d'avant le commencement, d'avant la naissance ${ }^{1}$.

Dans Cours au Bauhaus, il avait résumé leurs caractéristiques en deux colonnes :

\begin{tabular}{|l|l|}
\hline BLANC & NOIR \\
\hline \hline Résistance active, rebondissement & $\begin{array}{l}\text { Résistance passive, manque de résistance, } \\
\text { abandon }\end{array}$ \\
\hline \hline 1. Un mur sans fin & 1. Un trou sans fin \\
\hline \hline 2. Toutes les possibilités & 2. Sans issue \\
\hline \hline $\begin{array}{l}\text { 3. Lumière maximale : somme de tous les } \\
\text { rayons }\end{array}$ & 3. Obscurité absolue : sans rayonnement \\
\hline \hline 4. Sonorité la plus haute, inaudible & 4. Sonorité la plus grave : inaudible \\
\hline \hline 5. Naissance & 5. Mort \\
\hline \hline 6. Silence & Silence \\
\hline
\end{tabular}

Dans la deuxième moitié du XXe siècle, elle est en Amérique. Et en particulier au début des années cinquante, lorsqu'une volonté de jeunes artistes d'échapper à l'expressionnisme abstrait les pousse à mettre en œuvre un style de la retenue. Le monochrome, et au point extrême, le monochrome blanc, semble le lieu idéal de la négation :

« No texture, no brushwork or calligraphy, no sketching or drawing, no forms, no design, no colors, no light, no space, no time, no size or scale, no movement, no object, no subject, no matter $»^{3}$.

Cette evolution était reconnue par Lucy Lippard dans son article, « Silent Art » en 1967 :

«An exhibition of all-black paintings ranging from Rodchenko to Humphrey to Corbett to Reinhardt, or an exhibition of all-white paintings from Malevich to Klein, Kusama, Newman, Francis, Corbett, Martin, Irwin, Ryman and Rauschenberg would be a lesson to those who consider such art "empty" " 4 . 
Il semble aussi que ce soit la suite logique d'une évolution de l'art, moderniste et historicisée, telle qu'elle a été définie par Clement Greenberg. Par exemple, dans «Vers un nouveau Laocoon » publié dans Horizon en septembre 1940, il écrit :

" L'histoire de la peinture d'avant-garde est celle de sa soumission progressive aux lois de sa technique $»^{5}$,

ou dans « Modernist Painting », publié en 1960 dans Forum Lectures :

«L'essence du modernisme se fonde, selon moi, dans l'emploi des méthodologies distinctives d'une discipline pour critiquer cette discipline même, non pas pour la subvertir, mais pour l'ancrer plus fermement encore dans sa zone de compétence, »

ou

«Le modernisme a découvert que ces limites [conditions limites auxquelles une tableau doit se soumettre pour être encore perçu comme un tableau ] peuvent être repoussées indéfiniment, avant qu'un tableau cesse d'être un tableau pour devenir un objet arbitraire; mais il a aussi découvert que plus ces limites sont repoussées, plus elles doivent être respectées et soulignées de manière explicite $»^{6}$.

C'est évidemment le cas, tardif de Robert Ryman (fig. 23), qui dans ses commentaires sur ses oeuvres carrées et blanches souligne l'absence de symbolisme de ce choix, mais plutôt la volonté, en limitant le plus possible les choix, d'atteindre une sorte d'essence de la peinture: surface, épaisseur de la touche, remontée de couleurs sous-jacentes, support apparent, systèmes d'accrochage.

Une œuvre de Rauschenberg de 1953, Erased de Kooning Drawing (fig.19) constitue une transition parfaite avec l'idée que nous voulons développer ensuite. En effet, de prime abord, l'objectif transgressif de l'œuvre semble de transformer un De Kooning en une «non-image monochrome». De ce fait, la jeune génération efface symboliquement celle qui lui a précédé, tout en créant une nouvelle œuvre, en contradiction avec l'expressionnisme, une œuvre monochrome. Pourtant, par les traces encore lisibles du dessin effacé, c'est tout un autre univers que suggère cette œuvre : la projection des images du spectateur, l'insertion de l'imaginaire, qui substitue au rien l'infini possibilités de l'écran.

40 Cette idée de non-lieu doit beaucoup à une conférence que Christine Savinel, professeur à Paris III a donnée au Musée du Louvre en mars 2003: «Le cosmopolitisme de Whistler, Sargent, Wharton et James : une relation critique ${ }^{7}$. Elle y décrit le cosmopolitisme de la fin du XIXe siècle comme une sortie nécessaire du consensus identitaire que représentait la Hudson River School pour l'Amérique. Il n'est sans doute pas indifférent que ces deux peintres, ainsi que Mary Cassatt, la troisième grande expatriée, aient beaucoup utilisé la couleur blanche. Cette non-couleur renforce encore la création d'un non-lieu pictural. Chez Sargent, se joue comme un caché de son art, comme le rapport dialectique entre ses portraits mondains (où Christine Savinel remarque pourtant les fonds informels, voir fig. 8) et ses scènes de genre d'été, à la sensualité débridée (fig. 10). La désappropriation se fait par la représentation de non-lieux. La déréalisation du lieu permet l'avènement d'un lieu de l'art, toujours étrange, toujours flottant :

« Il est évident que cette déprise-là est celle de tout grand art qui sait qu'il ne peint que le rien et qu'elle ne passe pas forcément du tout par le cosmopolitisme. Mais pour la peinture américaine, celui-ci a probablement représenté le chemin obligé pour se dégager d'une double méprise, celle du réel et celle de l'identité nationale $»^{8}$. 
Curieusement, c'est aussi cette notion flottante, qui réapparaît au cœur des œuvres monochromes blanches des artistes américains des années 50. A ce propos, on peut prendre comme exemple les White Paintings que Rauschenberg réalise en 1951 (fig. 20). Ici, je m'appuie sur une excellent article de Branden Joseph, «Blanc sur blanc : Robert Rauschenberg et John Cage $»^{9}$. Il y cite la lettre de Robert Rauschenberg à Betty Parsons, le 18 octobre 1951, depuis le Black Mountain College sur les White Paintings :

"Ce sont de grandes toiles blanches (1 blanc pour 1 DIEU) que j'ai organisées et choisies avec l'expérience du temps et que je présente avec l'innocence d'une vierge. Elles traitent du silence organique, de son suspens, de son excitation et de son corps, de la contrainte et de la liberté de l'absence, de la plénitude plastique du rien, du point où naît et s'achève un cercle. Tout cela constitue une réponse naturelle aux pressions actuelles de ceux qui sont sans foi et c'est aussi une défense de l'optimisme intuitif. Que ce soit moi qui les faites n'a aucune importance - leur créateur, c'est Aujourd'hui ${ }^{10}$.

42 Si cette description n'ajoute au topos moderniste que la dimension spirituelle, c'est plus tard, à travers sa collaboration avec John Cage qu'il peut " échapper aux apories liées à une vision fondée sur la négation, et ouvrir une voie nouvelle au-delà du terminus de la peinture moderniste représenté par le monochrome ».

En effet, John Cage, dans un article qu'il consacre à son ami ${ }^{11}$, décrit ces œuvres de la manière suivante : « les White Paintings sont des aéroports pour les lumières, les ombres, les particules. »Cette idée qui évoque irrésistiblement la photographie du Grand Verre de Marcel Duchamp par Man Ray, Élevage de Poussière, permet paradoxalement à l'œuvre de Rauschenberg de prendre une nouvelle direction, le fait sortir de l'impasse d'une logique de réduction au médium, pour l'amener vers l'idée d'un écran, personnel à chaque spectateur, pour l'éphémère. De même, ce sont les tableaux blancs de Rauschenberg qui encourageront Cage à composer 4'33" de silence, un pièce où une seule note est tenue pendant cette durée, tandis que le spectateur est invité à écouter les bruits ambiants.

Il est difficile de parler de couleur en évitant la question des races. Pourtant, elle est souvent oubliée lorsque l'on parle du blanc. Comme si, la question des races ne se pose pas du point de vue de la race dominante, mais de la différence qui s'établit avec elle. Comme l'écrit Richard Dyer: «Une personne blanche qui parle ne reconnaît presque jamais qu'elle s'exprime depuis cette place et c'est sans doute ce qui définit la condition de blanc et son pouvoir : les blancs exigent et obtiennent l'autorité pour ce qu'ils disent en n'admettant pas, ou en ne prenant pas conscience, que, la plupart du temps, ils ne parlent que au nom de la blancheur ${ }^{12}$. Le blanc tire son pouvoir idéologique de sa transparence autoproclamée, de son auto-élévation au-dessus de la catégorie même de race. Pourtant, il ne devrait pas être impossible de lire également ce portrait par Sargent d'aristocrate anglais blond (fig. 8), à la peau diaphane et tout habillé de blanc, placé devant une colonne grecque, comme le renforcement par le portrait de l'idée de " crème de la crème ». Ceci est bien entendu à rapprocher d'une société coloniale, qui renforce sa position de blanc en l'opposant à celle des indigènes, en particulier par le vêtement.

On peut également remarquer que toute l'histoire de l'abstraction, pour universelle qu'elle semble dans la narration de l'histoire de l'art, a été produite par des blancs. 


\section{NOTES}

1. Cité dans Guila Ballas, La couleur dans la peinture moderne : théorie et pratique, Paris, Adam Biro, 1997, 227.

2. Ibid, 228.

3. Ad Rheinhardt, Twelve Rules for a New Academy, cité dans Carol Blotkamp, «Introduction », Basically White, London, Institute of Contemporary Arts, 1974, 7.

4. Lucy R. Lippard, «The Silent Art », Art in America, janvier-février 1967, 63.

5. " The history of avant-garde painting is that of a progressive surrender to the resistance of its medium [...]. », Clement Greenberg, " Vers un nouveau Laocoon », Horizon, septembre 1940, réimprimé dans The Collected Essays and Criticism, The University of Chicago Press, 1986, 34.

6. « The essence of Modernism lies, as I see it, in the use of characteristic methods of a discipline to criticize the discipline itself, not in order to subvert it but in order to entrench it more firmly in its area of competence, » et « Modernism has found that these limits [the limiting conditions with which a picture must comply in order to be experienced as a picture] can be pushed back indefinitely before a picture stops being a picture and turns into an arbitrary object ; but it has also found that the further back these limits are pushed the more explicitly they have to be observed and indicated, » in Clement Greenberg, « Modernist Painting », Forum Lectures, 1960, réimprimé dans The Collected Essays and Criticism, The University of Chicago Press, 1986, 85 et 89-90.

7. A paraître dans Matthias Waschek et Veerle Thielemans (ed.), L'Art américain. Identités d'une nation, Paris, Musée du Louvre, Terra Foundation for American Art, ENSBA, 2005, 84-102.

8. Ibid., 95-96.

9. Paru dans les Cahiers du Musée National d'Art Moderne, no. 71, printemps 2000, 4-31.

10. Ibid., 6.

11. John Cage, « On Robert Rauschenberg, Artist, and His Work », Silence, Middletown (Conn.), Wesleyan University Press, 1961, 102.

12. Richard Dyer, White, London, Routledge, 1997, xiv. 